\title{
THE IMPACT OF LIGHT AND GRAVITY ON GROWTH DIRECTIONS IN A ROOT SYSTEM OF CUCUMIS SATIVUS L.
}

\author{
PIOTR OTRĘBA \\ Institute of Plant Biology, University of Wrocław \\ Kanonia 6/8, 50-328 Wrocław, Poland \\ e-mail: pio-o@pio-o.net
}

(Received: October 24, 2008. Accepted: January 23, 2009)

\begin{abstract}
While each individual root responds to such environmental factors as light or gravity the question arises how these reactions subordinate to the whole root system, which is supposed to maintain its primary functions. Data presented here confirm that in cucumber the gravity and light modulate the growth direction of the lateral roots subunits of the system. Another important factor affecting behavior of lateral roots is an orientation of the main root. These facts all together suggest that the root system functions as an integrated entity, capable of adapting its architecture to changing environmental conditions. Its flexibility, based on unknown signaling network, guarantees optimal functioning of the system.
\end{abstract}

KEY WORDS: gravitropic setpoint angle, gravitropism, phototropism, lateral roots, Cucumis sativus.

\section{INTRODUCTION}

Plant architecture results from oriented growth of plant organs in 3D space. This process, which is precisely controlled on the whole plant (genet) level by intrinsic mechanisms, assuring proper response of subunits (ramets) to changing environmental conditions, requires integration of many internal and external signals. Among the latter, the most important are gravity and light.

It is known that plant organs, such as stems or roots, growing in steady state condition, acquire and maintain a definite and stable orientation with respect to the gravity vector. This orientation, called the gravitropic setpoint angle (GSA) depends upon the type and developmental stage of the organ, thus it may change in the course of ontogeny. It can also be modified by environmental factors such as light or gravistimulation by reorienting the plant (Digby and Firn 1995; Firn and Digby 1997). It has been arbitrarily decided, that a plant organ growing downwards (for example a main root) has GSA of $0^{\circ}$, whereas an organ growing upwards (like a stem) has GSA of $180^{\circ}$. Lateral organs acquire GSA of intermediate values (Digby and Firn 1995).

In course of their evolutionary history plants developed special systems responsible for the perception of a given environmental stimulus (Hangarter 1997; Blancaflor et al. 1998; Chen et al. 1999; MacCleery and Kiss 1999; Kiss et al. 2002; Wolverton et al. 2002; Kiss et al. 2003a). Changes in quality, quantity or direction of the acting stimulus may affect the growth direction of a particular plant organ adapting it to the new altered conditions. If the response is achieved by differential growth rates at the opposite flanks of a plant organ, this reaction is called a tropism (Srivastava 2002). The tropisms are categorized according to the kind of stimulus involved, e.g. gravitropism, phototropism, hydrotropism, i.e. a growth response with respect to the gravity vector, to the light source and to the moisture, respectively (Chen et al. 1999; Kiss et al. 2002; Kiss 2007). The plant organ may grow towards the stimulus exhibiting a positive tropism or away from it showing a negative one. Thus, the tropisms may be regarded as a tool to render or acquire the specific GSA by the growing plant organs (Digby and Firn 1995).

The primary role of the entire root system is to anchor a plant to the ground and to supply it with water and nutrients (apart from other additional functions, such as e.g. storing). As a consequence, the root system develops the appropriate architecture, which is mostly species specific and may depend on the habitat conditions (Taiz and Zeiger 2002). There are generally recognized two main types of the belowground root systems in higher vascular plants: a) taproot system - characteristic of gymnosperms and Dicotyledones; b) fibrous root system - present in Monocotyledones (Taiz and Zeiger 2002). The main root in taproot system or inner adventitious roots of fibrous root system can be characterized as having GSA ca. $0^{\circ}$. GSA of the lateral roots (taproot system) or outer roots (fibrous root system) ranges from $45^{\circ}$ to $90^{\circ}$, at least in the initial stages of deve- 
lopment. Usually, the GSA decreases during further growth, that means their growth direction becomes closer to $0^{\circ}$ (Firn and Digby 1997; Kiss et al. 2002). It has been postulated that this developmental change of the growth direction may be associated with maturation of the statocytes, the specialized plant cells involved in gravisensing. More precisely, cellular components such as amyloplasts, endoplasmic reticulum (ER) and its specific form - nodal $\mathrm{ER}$, as well as the intracellular distribution of these organelles are the most important factors being considered (Zheng and Staehelin 2001; Kiss et al. 2002; Kuya et al. 2006). Additionally, establishing and maintenance of the given growth direction may be under the genetic control (Mullen and Hangarter 2003). In general, roots are sensitive to both gravity and light, even though that light can hardly penetrate the ground (Mandoli et al. 1990). Gravitropism is more significant in root growth than phototropism playing the crucial role in establishing the root system architecture (Kiss et al. 2002). Nevertheless, light modulates the gravitropic response of the roots. Depending on its wavelength or location of its source, light can strengthen positive gravitropic response (blue or white light) or reduce it (red light or illumination of the roots from the bottom with blue or white light (Correll and Kiss 2002; Kiss et al. 2002; Kiss et al. 2003b). Considering the function of the root system, its resultant architecture cannot be accidental.

The major questions rising in this context are following: does the architecture of the root system adjust to such affecting factors as reorientation or light to preserve its primary function? Is the reaction of the whole root system integrated? Or is it simply the sum of individual root reactions? How the change of the environmental factors affects the GSA of the entire system, especially lateral roots? This paper was inspired by these questions.

\section{MATERIALS AND METHODS}

The analysis of the root system architecture was performed on the seedlings of Cucumis sativus L. varietas Wisconsin grown in vitro. Cucumber seeds were sterilized with $5 \%$ sodium hypochlorite and $70 \%$ ethanol. After rin-

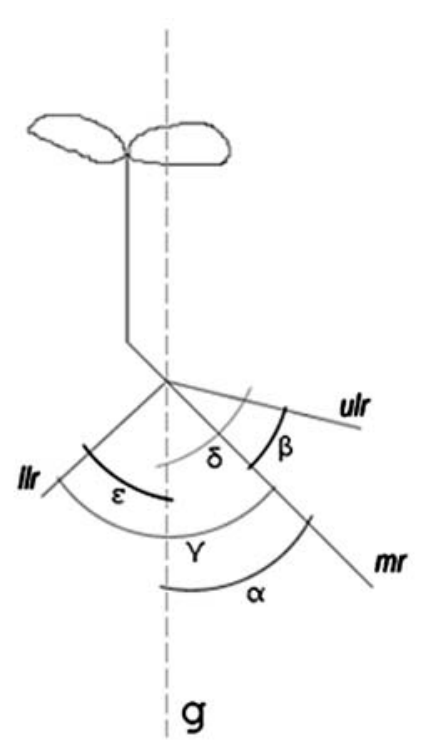

Abbreviations: $\mathrm{mr}$ - main root; ulr - upper lateral root; 1lr - lower lateral root; g - gravity vector; $\alpha$ - the angle of the main root to the gravity vector; $\beta$ and $\gamma-$ the angles at which the lateral roots depart from the main root $(\beta+\gamma-$ the angle between two lateral roots emerging at the same level opposite one to another); $\delta$ and $\varepsilon-$ the angles of the lateral (ulr and llr, respectively) roots with respect to the gravity vector. sing few times in distilled water, they were placed on the standard Hoagland nitrate medium $\mathrm{pH}$ 6.4-6.5 solidified with Phytagel (Sigma - Aldrich), which is transparent enough to observe the roots. The plants grew for up to 14 days in closed glass bottles in the presence of light (16 hours day/6 hours night) or in continuous darkness. In both combinations the temperature was $27^{\circ}$.

To examine the lateral root reaction to changing light conditions, the seedlings with well developed root system were either exposed to light or moved to darkness.

The initial state of the system as well as its response to the treatment had been quantified with use of parameters $\alpha$, $\beta, \gamma, \delta$ and $\varepsilon$, explained in Figure 1. When the main root grew at the angle bigger than $20^{\circ}$ with respect to the gravity vector, the lateral roots were distinguished as lower and upper ones, based on the side of their emergence (Fig. 1). The root system pictures, taken with digital camcorder Sony DCR-PC350E, were subsequently processed with Fireworks MX 2004, Adobe. These images had been used for measuring $\alpha, \beta, \gamma$ values, with the help of AutoCAD2000, Autodesk.

TABLE 1 . The parameters of root system architecture $(\alpha, \beta$ and $\gamma)$ in 10 plants with almost vertical main root, growing in the presence of light. All data are given in degrees. Each horizontal line refers to the single root or to a pair of lateral roots emerging from the main root at the same level, each column, for a given plant, refers to the parameters of consecutive lateral roots in their developmental sequence: downward from older (proximal) to younger (distal) ones.

\begin{tabular}{|c|c|c|c|}
\hline Plant $\mathrm{N}^{\circ}$ & $\alpha$ & $\beta$ & $\gamma$ \\
\hline \multirow[t]{9}{*}{1} & 6 & 68 & \\
\hline & 6 & 64 & 65 \\
\hline & 6 & 77 & 68 \\
\hline & 6 & 77 & 75 \\
\hline & 6 & 82 & \\
\hline & 6 & 61 & 58 \\
\hline & 6 & 70 & 61 \\
\hline & 6 & 77 & 78 \\
\hline & 6 & 52 & 68 \\
\hline \multirow[t]{2}{*}{2} & 6 & 62 & 51 \\
\hline & 6 & 76 & 71 \\
\hline \multirow[t]{3}{*}{3} & 4 & 75 & 68 \\
\hline & 4 & 76 & 70 \\
\hline & 0 & 72 & 81 \\
\hline 4 & 15 & 89 & 58 \\
\hline \multirow[t]{2}{*}{5} & 13 & 72 & 47 \\
\hline & 13 & 67 & 41 \\
\hline \multirow[t]{4}{*}{6} & 18 & 86 & 59 \\
\hline & 18 & 95 & 68 \\
\hline & 10 & 62 & \\
\hline & 10 & 73 & \\
\hline \multirow[t]{3}{*}{7} & 5 & 66 & 53 \\
\hline & 5 & 59 & 63 \\
\hline & 8 & 71 & 74 \\
\hline \multirow[t]{3}{*}{8} & 2 & 43 & 41 \\
\hline & 2 & 55 & 44 \\
\hline & 7 & 67 & 66 \\
\hline \multirow[t]{3}{*}{9} & 4 & 45 & \\
\hline & 4 & 61 & 46 \\
\hline & 3 & 49 & 45 \\
\hline \multirow[t]{7}{*}{10} & 15 & 68 & 64 \\
\hline & 9 & 74 & 63 \\
\hline & 0 & 57 & 65 \\
\hline & 16 & 71 & 56 \\
\hline & 16 & 87 & 55 \\
\hline & 16 & 48 & \\
\hline & 3 & 78 & \\
\hline
\end{tabular}

Fig. 1. Scheme of the root system architecture. 
TABLE 2. The parameters of root system architecture $(\alpha, \beta$ and $\gamma)$ in 8 plants with slanted main root, growing in the presence of light. $\beta$ and $\gamma$ values refer to the upper and the lower lateral roots respectively. Further description as in Table 1. Two additional angles were measured here $-\delta$ (the angle between upper lateral roots and gravity vector) and $\varepsilon$ (the angle between lower lateral roots and gravity vector).

\begin{tabular}{|c|c|c|c|c|c|}
\hline Plant $\mathrm{N}^{\circ}$ & $\alpha$ & $\beta$ & $\gamma$ & $\delta(\beta+\alpha)$ & $\varepsilon(\gamma-\alpha)$ \\
\hline \multirow[t]{2}{*}{1} & 24 & 51 & 92 & 75.0 & 68.0 \\
\hline & 24 & 66 & 90 & 90.0 & 66.0 \\
\hline 2 & 27 & 46 & 90 & 73.0 & 63.0 \\
\hline \multirow[t]{3}{*}{3} & 25 & & 86 & & \\
\hline & 25 & 54 & 93 & 79.0 & 68.0 \\
\hline & 25 & 62 & 89 & 87.0 & 64.0 \\
\hline \multirow[t]{4}{*}{4} & 51 & 47 & 86 & 98.0 & 35.0 \\
\hline & 51 & 46 & 79 & 97.0 & 28.0 \\
\hline & 82 & 51 & 110 & 133.0 & 28.0 \\
\hline & 82 & 34 & 87 & 116.0 & 5.0 \\
\hline \multirow[t]{2}{*}{5} & 88 & & 106 & & \\
\hline & 88 & 62 & & 150.0 & \\
\hline \multirow[t]{3}{*}{6} & 26 & & 97 & 26.0 & \\
\hline & 26 & & 101 & 26.0 & \\
\hline & 27 & 59 & 95 & 86.0 & 68.0 \\
\hline \multirow[t]{2}{*}{7} & 74 & 20 & 99 & 94.0 & 25.0 \\
\hline & 21 & 45 & 66 & 66.0 & 45.0 \\
\hline 8 & 68 & & 74 & & \\
\hline
\end{tabular}

TABLE 3. The parameters of root system architecture $(\alpha, \beta$ and $\gamma)$ in 8 plants with almost vertical main root, growing in absence of light. Further description as in Table 1.

\begin{tabular}{|c|c|c|c|}
\hline Plant $\mathrm{N}^{\circ}$ & $\alpha$ & $\beta$ & $\gamma$ \\
\hline \multirow[t]{4}{*}{1} & 9 & 107 & 81 \\
\hline & 9 & 110 & 116 \\
\hline & 6 & 90 & \\
\hline & 6 & 94 & \\
\hline \multirow[t]{5}{*}{2} & 19 & 104 & 82 \\
\hline & 19 & 111 & 84 \\
\hline & 19 & 110 & 95 \\
\hline & 19 & 104 & 93 \\
\hline & 6 & 96 & 82 \\
\hline 3 & 14 & 85 & 95 \\
\hline \multirow[t]{3}{*}{4} & 12 & 101 & \\
\hline & 12 & 86 & \\
\hline & 7 & 92 & \\
\hline 5 & 19 & 91 & 85 \\
\hline 6 & 0 & 102 & 84 \\
\hline \multirow[t]{5}{*}{7} & 16 & 90 & 88 \\
\hline & 16 & 70 & 98 \\
\hline & 15 & 101 & 74 \\
\hline & 20 & 88 & 85 \\
\hline & 20 & 85 & \\
\hline 8 & 2 & 88 & 100 \\
\hline
\end{tabular}

\section{RESULTS}

The first lateral roots developed in 5 to 8 days after seed sowing. Lateral roots usually emerged in pairs at opposite flanks of the main root. Single roots appeared but rarely (sporadically).

The growth direction of the main root tended to be parallel to the gravity vector. However, the individual main roots often changed their growth course (in steady state) thus their angle to the gravity vector $(\alpha)$ was sometimes different at the basal and at the distal region of the root.

\section{Root system}

of the plants growing in the presence of light

From the total of 18 plants the main root of 10 plants assumed almost vertical growth. Deviations from the gravity vector $(\alpha)$, ontogenetically increasing or decreasing, had never exceeded $20^{\circ}$ (Table 1$)$. Lateral roots departed from the main roots at the angle ( $\beta$ or $\gamma$ ) averaging $65^{\circ}$ (Fig. $2 a$, c). The values of $\beta$ and $\gamma$, in most of the roots emerging in pairs, were similar. They were noticeably different in cases when $\alpha$ was higher than $10^{\circ}$. Average of added $\beta$ and $\gamma$ values, for all the root pairs, was $130^{\circ}$.

In the remaining 8 plants $\alpha$ parameter exceeded $20^{\circ}$ (Table 2). With this inclination of the main root, two sets of lateral roots could be distinguished - upper and lower (Figs 1 and $2 \mathrm{e}$ ). The average value of the angle $\beta$, at which the upper lateral roots departed from the main root, was $49^{\circ}$ while the average value of the angle $\gamma$ for lower lateral roots was $91^{\circ}$ (Table 2). Average of added $\beta$ and $\gamma$ values for all the root pairs was $138^{\circ}$, not very different from the value calculated for plants with the main roots vertical.

Root system

of the plants growing in darkness

In this experiment 17 plants were examined. As previously, they had been divided into two groups depending upon the range of $\alpha$ parameter. For 8 plants $\alpha$ varied between $0^{\circ}$ and $20^{\circ}$ (Table 3 ). Characteristically the lateral roots departed from the main root at the right angle (Figs 2b, d).

For 9 plants the $\alpha$ parameter was bigger than $20^{\circ}$ (Table 4 , Fig. $2 \mathrm{f}$ ). The angle $\beta$ of the upper lateral roots was about

TABLE 4. The parameters of root system architecture $(\alpha, \beta$ and $\gamma)$ in 9 plants with slanted main root, growing in absence of light. Further description as in Table 1.

\begin{tabular}{|c|c|c|c|}
\hline Plant $N^{\circ}$ & $\alpha$ & $\beta$ & $\gamma$ \\
\hline \multirow[t]{4}{*}{1} & 63 & 94 & 59 \\
\hline & 21 & & 70 \\
\hline & 21 & 97 & 93 \\
\hline & 21 & 108 & 96 \\
\hline 2 & 21 & 101 & 87 \\
\hline \multirow[t]{7}{*}{3} & 29 & 98 & 75 \\
\hline & 29 & & \\
\hline & 29 & 94 & 90 \\
\hline & 29 & 100 & \\
\hline & 39 & 97 & 87 \\
\hline & 39 & & 76 \\
\hline & 39 & 98 & 75 \\
\hline \multirow[t]{2}{*}{4} & 41 & 98 & 75 \\
\hline & 41 & 119 & 89 \\
\hline \multirow[t]{5}{*}{5} & 36 & 94 & 71 \\
\hline & 36 & 95 & 67 \\
\hline & 36 & 115 & \\
\hline & 29 & & \\
\hline & 29 & 103 & 61 \\
\hline \multirow[t]{4}{*}{6} & 68 & 109 & 60 \\
\hline & 68 & & \\
\hline & 68 & & 67 \\
\hline & 68 & 88 & 81 \\
\hline \multirow[t]{2}{*}{7} & 20 & 85 & 85 \\
\hline & 20 & 102 & \\
\hline 8 & 27 & 102 & 84 \\
\hline \multirow[t]{3}{*}{9} & 26 & 78 & 80 \\
\hline & 38 & 97 & 78 \\
\hline & 50 & & 51 \\
\hline
\end{tabular}


$76^{\circ}$. As in previous experiment, the angle $\gamma$ for lower lateral roots was bigger than $\beta$, averaging $99^{\circ}$ (Table 4 ). Compared to the case of plants grown in the light the difference between $\beta$ and $\gamma$ was much less pronounced. The average sum of $\beta$ and $\gamma$ angles for all the root pairs was close to $180^{\circ}$, similarly as in plants with vertical roots (Tables 3 and 4).

\section{Growth response}

of the root system to changing light conditions

For five plants examined the growth direction of the lateral roots rapidly changed in the plants, initially grown in the darkness and suddenly exposed to light. Their GSA characteristically decreased (Figs $2 \mathrm{~g}$, h). The growth of some lateral roots was entirely inhibited by the treatment.

Moving plants grown in light conditions to the darkness (five plants examined) brought up opposite effects: their la- teral roots acquired new GSA of the higher value in a process of slow and continuous change. This time the change in growth direction and GSA was slow but continuous (Fig. 2i).

\section{Growth response}

of the root system to reorientation in the darkness

For five plants examined the growth direction of the lateral roots rapidly changed in the plants that undergone reorientation in the darkness (Figs $2 \mathrm{j}, \mathrm{k}$ ). They acquired smaller GSA in comparison to the state before reorientation. This shows that even if the growth direction of lateral root occurs horizontally, they are capable of perceiving and reacting to changing orientation vs gravity vector.
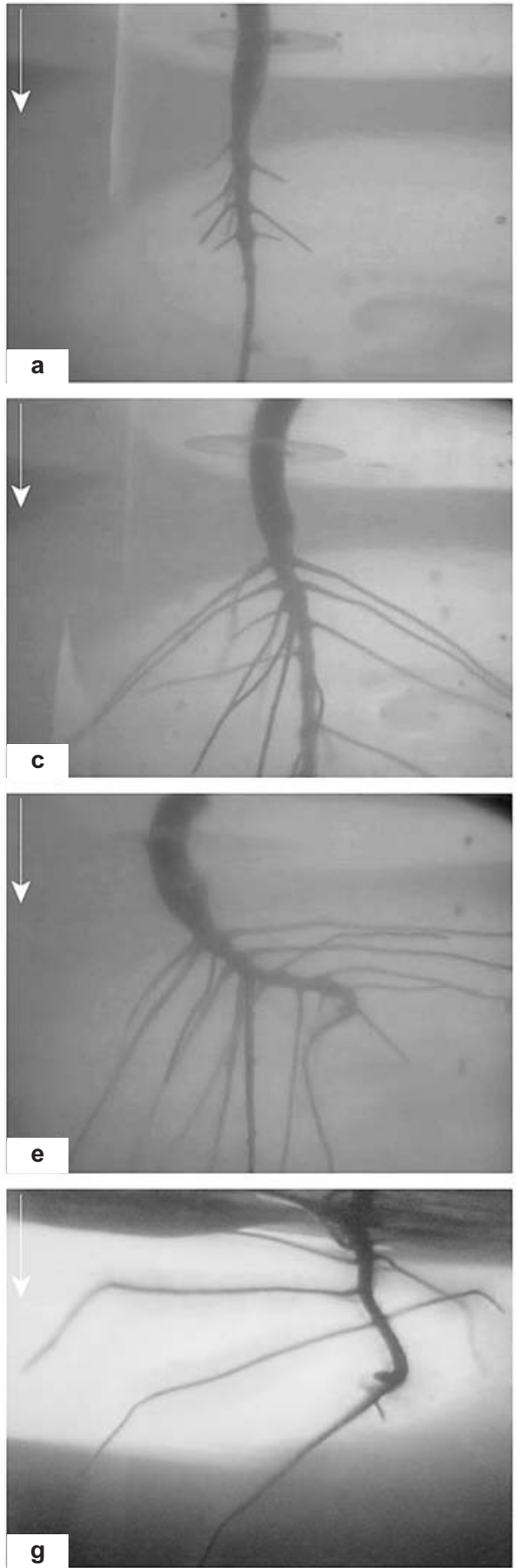
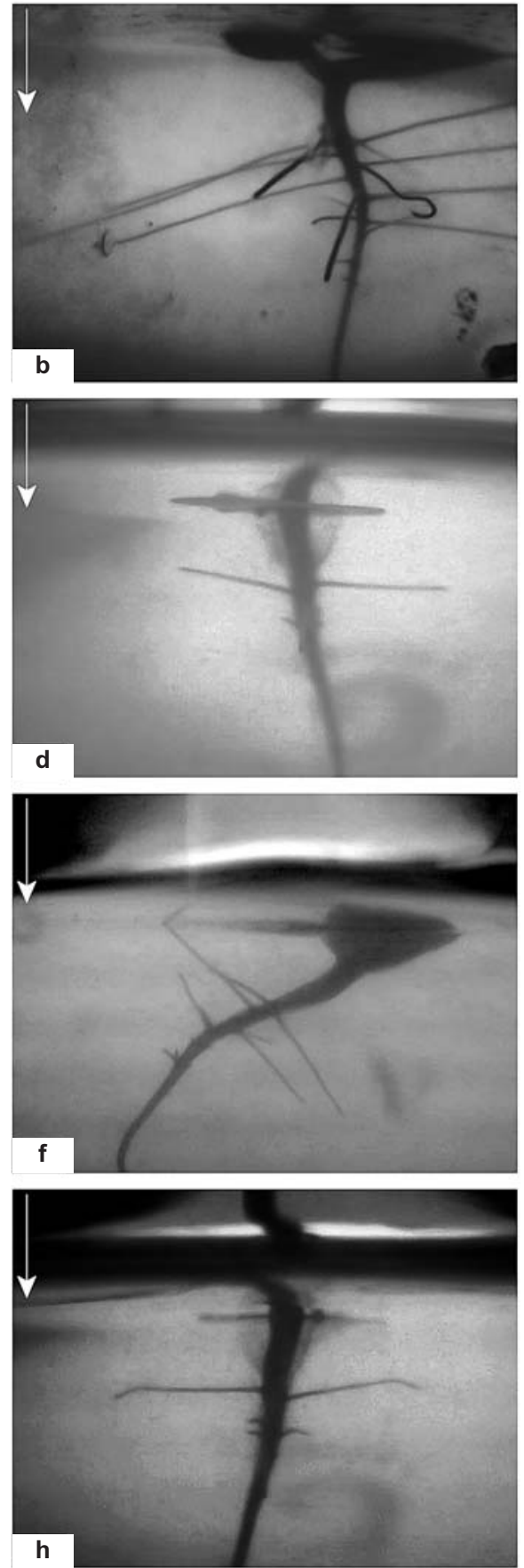

Fig. 2. The root system architecture of $\mathrm{Cucu}$ mis sativus in different light conditions: a, c, $\mathrm{e}-$ plants grown in the presence of light (long-day); b, d, f - grown in continuous darkness; $\mathrm{g}, \mathrm{h}-$ plants moved from darkness to light; i - plants moved from light to darkness. In figures a-d the main root is oriented \pm parallel to the gravity vector (at angle less than $20^{\circ}$ ), e-f the main root is clearly inclined. In figures $\mathrm{j}, \mathrm{k}$ part of the root system of the plant grown in the darkness is present; after lateral root (marked with thick white arrow) achieved about $15 \mathrm{~mm}$ (j) the plant was reoriented $(\mathrm{k})$ - a change of lateral root growth direction is observed (k). In all images a thin arrow indicates the gravity vector $(\mathrm{g})$. 


\section{DISCUSSION}

According to the recent literature, as it has been recently reported, the characteristic growth pattern of the lateral roots manifested by their initially horizontal then declined downwards growth is associated with development and maturation of statocytes (Kiss et al. 2002; Kuya et al. 2006). Kiss et al. (2002) have shown a correlation between positive gravitropism and development of amyloplasts in columella cells of $A$. thaliana lateral roots suggesting that large mature amyloplasts are required for the lateral roots to be sensitive to the gravity. However, Kuya et al. (2006) have recently demonstrated that amyloplasts can react to gravity stimulus in the initial stage of the lateral root development but this response does not trigger the change of the growth direction. These results indicate that possibly other factors apart from amyloplasts are involved in regulation of the lateral root system orientation. Data presented here showed that lateral roots maintained their growth direction while grown in stable light conditions (Figs 2a, f). It may be a consequence of the developmental stage of statocytes, as shown in the mentioned above research. At the same time, the lateral roots growing in darkness, after reorientation (gravistimulation) exhibited a change in the growth direction (Mullen and Hangarter 2003; Fig. 2k). This fact indicates the competence of these lateral roots to perceive the gravity stimulus. The question arises, however, why, in spite of being competent to the stimulus, the lateral roots, which have not been gravistimulated, continue the growth without the change of orientation. It may be suggested that lateral roots maintain the programmed direction of the growth, what appears to be consistent with the GSA concept (Digby and Firn 1995), although the precise mechanism involved still remains unknown. The GSA of the lateral roots is considerably affected by light (Digby and Firn 1997) as it was confirmed also in present studies. Depending on the light conditions applied, the growth reaction significantly differed with the rapid decrease of the GSA after exposure to light (Figs $2 \mathrm{~g}$, h) and with the gra- dual increase of the GSA of the lateral roots in plants moved to darkness (Fig. 2i). It seems that, independently of the current growth direction, after the change of light conditions the lateral roots try to reestablish the GSA typical of the given light conditions. An interesting tendency to maintain the angle value between the pair of lateral roots (Figs 2a, d) was observed in the intact, not reoriented and growing in stable light condition root system. This phenomenon also occurred when the orientation of the main root differed from vertical (Figs 2e, f). In extreme cases, such lateral roots grew almost vertically upwards, but the angle value between the pair of opposite lateral roots was comparable (or even) to this value in the system with a normal vertically oriented main root. As such a behavior of the root system was observed exclusively in darkness, it might suggest that a principle governing the architecture of the root system is a reciprocal orientation of the main and lateral roots and/or programmed angle between the opposite lateral roots. Light modulates this growth response. Plants that grew in the presence of light and had a declined main root provide us with especially interesting data on the root architecture influenced by light. The upper lateral roots of these plants underwent greater stimulation, acquiring a smaller value of the angle with respect to the main root (in comparison to plants growing in darkness), whereas the angles of the lower roots remained similar. Additionally, interesting similarities between angles $\beta$ to $\varepsilon$ and $\gamma$ to $\delta$ (Fig. 1) were observed indicating some geometrical nature governing the architecture of the root system, which needs further investigation. First of all, it may suggest that the reaction to light is not the "all or nothing" type because only one set of lateral roots shows the change in the growth pattern. Secondly, light offers additional information for upper lateral roots on what growth direction should be avoided. Taken together, it makes possible to maintain the primary function of the root system.

In conclusion, lateral roots represent an interesting model to investigate interactions between environmental stimuli and the resultant plant architecture. Data presented here in-
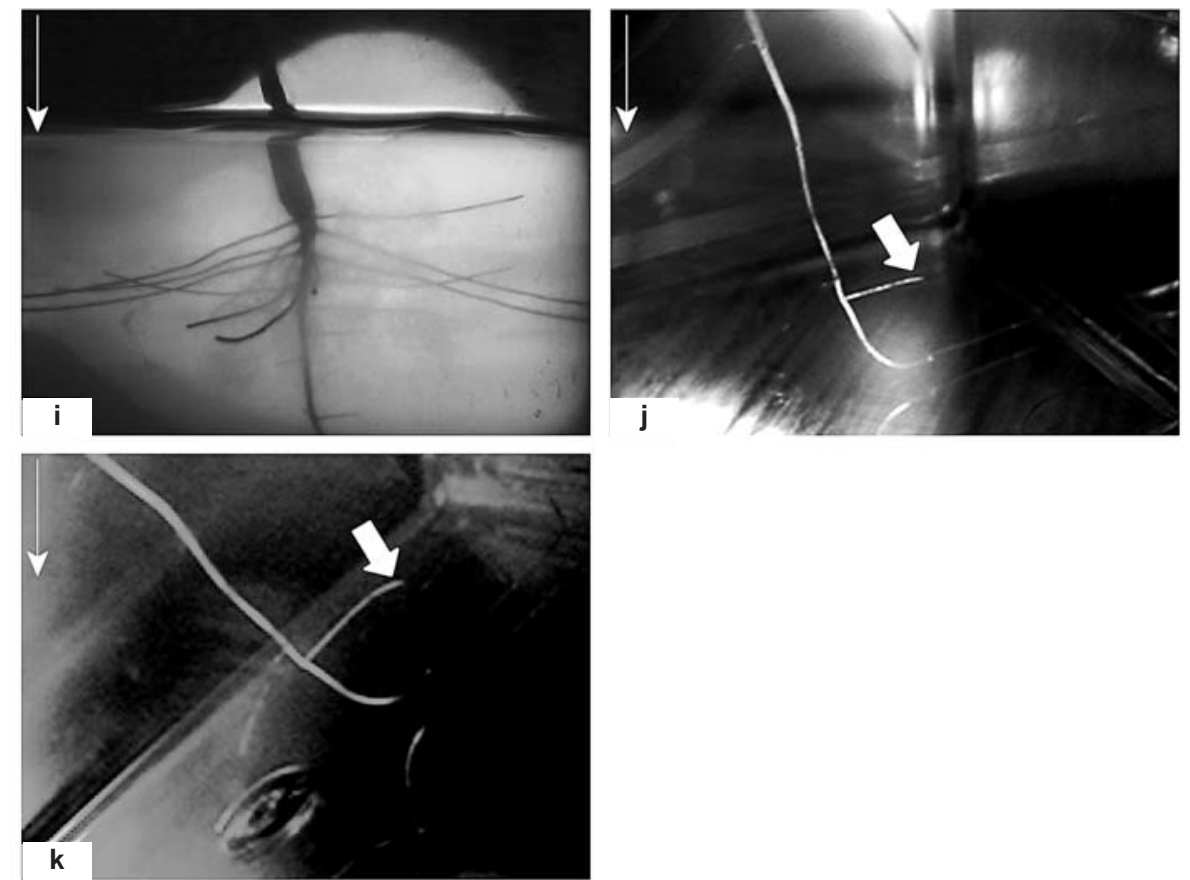
dicate that to perform its task the root system adjusts precisely to affecting external factors not at random manner. The root system architecture reveals an integrated response of all individual roots that have intrinsic mechanisms to react properly to changing environment but at the same time the prevalent remains the primary function of the entire system. It may be achieved by the complex control of growth processes at organismal level.

\section{ACKNOWLEDGEMENTS}

I would like to thank Prof. Beata Zagórska-Marek for discussion and helpful suggestions.

\section{LITERATURE CITED}

BLANCAFLOR E.B., HASENSTEIN K.H. 1997. The organization of the actin cytoskeleton in vertical and graviresponding primary roots of maize. Plant Physiol. 113: 1447-1455.

CHEN R., ROSEN E., MASSON P. 1999. Gravitropism in higher plants. Plant Physiol. 120: 343-350.

CORRELL M.J., KISS J.Z. 2002. Interactions between gravitropism and phototropism in plants. J. Plant. Growth Regul. 21: 89-101.

DIGBY J., FIRN R.D. 1995. The gravitropic set-point angle (GSA): the identification of an important developmentally controlled variable governing plant architecture. Plant Cell Environ. 18: 1434-1440.

FIRN R.D., DIGBY J. 1997. Solving the puzzle of gravitropism: has a lost piece been found? Planta 203: 159-163.

HANGARTER R.P. 1997. Gravity, light and plant form. Plant Cell Environ. 20: 796-800.

KISS J.Z., MILLER K.M., OGDEN L.A., ROTH K.K. 2002. Phototropism and gravitropism in lateral roots of Arabidopsis. Plant Cell Physiology 43: 35-43.
KISS J.Z., MULLEN J.L., CORRELL M.J., HANGARTER R.P. 2003. Phytochromes A and B mediate red - light - induced positive phototropism in roots. Plant Physiology 131: 1411$-1417$.

KISS J.Z., CORRELL M.J., MULLEN J.L., HANGARTER R.P., EDELMANN R.E. 2003. Root phototropism: how light and gravity interact in shaping plant form. Gravit. Space Biol. Bul. 16(2): 55-60.

KISS J.Z. 2007. Where's the water? Hydrotropism in plants. PNAS 104, 11: 4247-4248.

KUYA N., SATO Y., KANETA T., SATO S. 2006. Comparative study of the magnitude of gravitropism and the features of statocytes in primary and lateral roots of Adzuki bean. J. Plant Res. 119: 81.

MACCLEERY S., KISS J.Z. 1999. Plastid sedimentation kinetics in roots of wild-type and starch-deficient mutants of Arabidopsis. Plant Physiology 120: 183-192.

MANDOLI D.F., FORD G., WALDRON L., NEMSON J., BRIGGS W.R. 1990. Some spectral properties of several soil types: implications for photomorphogenesis. Plant Cell Environ. 13: 287-294.

MULLEN J.L., HANGARTER R.P. 2003. Genetic analysis of the gravitropic set-point angle in lateral roots of Arabidopsis. Adv. Space Res. 31(10): 2229-2236.

SRIVASTAVA L.M. 2002. Plant Growth and Development. Hormones and Environment. Academic Press, Amsterdam, Boston, London, New York, Oxford, Paris, San Diego, San Francisco, Singapore, Sydney, Tokyo.

TAIZ L., ZEIGER E. 2002. Plant Physiology. 3rd ed. Sinauer Associates, Inc. Publishers. Sunderland, MA.

WOLVERTON J., MULLEN J.L., ISHIKAWA H., EVANS M.L. 2002. Root gravitropism in response to a signal originating outside of the cap. Planta 215: 153-157.

ZHENG H.Q., STAEHELIN L.A. 2001. Nodal endoplasmic reticulum, a specialized form of endoplasmic reticulum found in gravity - sensing root tip columella cells. Plant Physiol. 125: 252-265. 